\title{
Real Terrain Modeling and Application Based on DOM Wang Yongsheng ${ }^{1,{ }^{,}{ }^{,},}$, Liu Xiao ${ }^{2, b}$ and Zhang Qizhi ${ }^{2, c}$ \\ ${ }^{1}$ School of art and design, Lanzhou Jiaotong University, Lanzhou 730070, China \\ ${ }^{2}$ School of art and design, Lanzhou Jiaotong University, Lanzhou 730070, China \\ awys_Iz@126.com, beiio@sina.com, ${ }^{\text {c}}$ zhangqizhi1990@126.com
}

\begin{abstract}
When creating artificial 3D natural scene, the terrain is complicated and working quantity is huge, relying on traditional modes for field investigation, mapping and modeling usually needs to consume rather quantity of manpower, material resource, financial resources and vigor. Rapid interception method is studied through concerted effort coordination of various 3D software by taking high-resolution Digital Orthophoto Map (DOM) in Liancheng, Yongdeng, Lanzhou, Gansu shown in Google Earth for an example. The purpose is to seek for transformation from various complicated terrains to digital data document and transformation from data document to 3D model and to reproduce non-destructive digitization 3D virtualization of real terrain rapidly and visually.
\end{abstract}

Keywords: Digital Orthophoto Map (DOM), Digital Elevation Model (DEM), Geographic Information System (GIS), Google Earth.

\section{Introduction}

DOM refers to Digital Orthophoto Map based on the monochrome or colored airphoto or remote-sensing image that has been scanned and digitally processed (it is directly acquired in digital mode). each pixel of the existed DEM data experiences radiation correction, differential rectification and digital mosaic, and then is clipped as per the topographic map limit of national principal scale, transformed to high-precision visual real image data unit integration, overlaid on the image plane in the form of km grid, mapborder finishing and annotation and generates image database saved in the form of grid data. DOM has many advantages such as high precision, rich information, perceptual intuition and strong reality. It can be applied to revision of topographic map, fabrication of composite digital product and 3D landscape map, general exploitation of land utilization and dynamic surveying in urban planning and design, land utilization database building and updating, dynamic monitoring of territorial resources and environment and GIS background information.

$\mathrm{Lu}$ Tusi governance of Liancheng, located at $65 \mathrm{~km}$ southwest of Yongdeng, Lanzhou, is a completely reserved ancient palatial building complex in various Tusi buildings at the border of Qinghai. The building complex is made up of Lu Tusi Governance, Miaoyin Temple and Tusi Garden. It is Major National Historical and Cultural Sites in territory of Gansu. The method can be used for the implementation of 3D modeling of Lu Tusi Governance. At present, in the major protection and inheritance of such historic scene, relic and culture relic unit, utilization rapid digital means can show the natural and real terrain rapidly so as to save a large quantity of manpower and material resources.

\section{DOM data extraction}

\subsection{Software environment erection}

Google Earth 6.1.0: Google Earth is a high-resolution virtual earth software developed by Google Inc. It integrates DOM of satellite remote sensing and aerial image and GIS covering physical relief DEM data and lists them in a virtual earth 3D model. 
3D Ripper DX v1.8: it is a window tool, used for intercepting 3D software model based on DirectX. The intercepted model is exported in the format of 3DR and then imported to 3ds max 2011 for next edition.

Imported 3DR file plug-in 3DRimport.dli in 3ds max 2011 version: after installation, increase the function of 3ds max 2011 in reading file in 3DR format.

3ds Max 2011: it is one of common 3D software, used for importing the intercepted 3DR file of 3D terrain model and imported it to OBJ format file.

Deep Exploration 6.3: it is 3D file conversion software. It can convert various files of 3D design software into compatible format so as to build a bridge for communication of different software. The software is used in this research to transform OBJ format to C4D format file containing texture and chartlet.

Cinema 4D R17: the software is easily used professional 3D software. It can be used freely in 3D animation, graph, special effect, visualization or rendering and thus called professional 3D kit of 3D designer. It can optimize the intercepted 3D terrain model and import it in a common format for further manufacturing.

\subsection{Data Extraction process}

\subsubsection{Initialization setting of software environment}

Firstly, we initialize Google Earth. After opening Google Earth for the first time, click "tool” in menu bar and select "option..." in the pull-down menu. As shown in Figure 1, select "DirectX" for "graph mode" in the "Google Earth Option” Dialog Box. Then, close "Google Earth Option” Dialog Box and close Google Earth. In this way, we complete initialization setting.

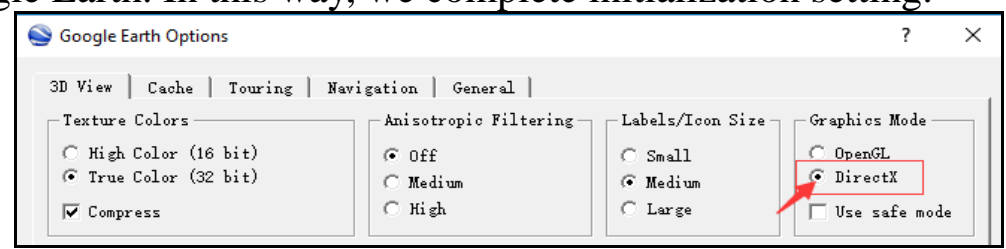

Fig. 1 Option setting of Google Earth

Secondly, we initialize 3D Ripper DX v1.8. After opening 3D Ripper DX v1.8, as shown in Figure 2, input main program path of Google Earth in "Please select DirectX 6/8/9 application to analize" on the top of dialog box of setting popped up and import Frames, Textures and Shaders to "Frames (*.3DR) output directory”, “Textures (*.DDS) output directory” and "Shaders (*.VSH,*.PSH) output directory” and save it in a drive with big memory space. Other settings are default.

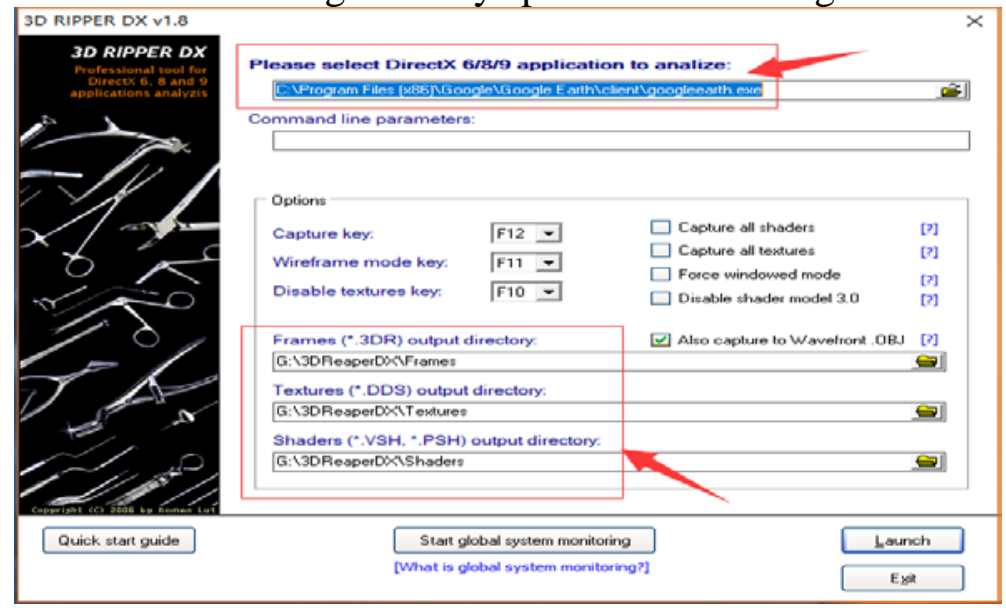

Fig. 2 Setting of 3D Ripper DX v1.8

At last, in the 3D Ripper DX v1.8 setting dialog box with initialized environment is set, click "Launch" to start Google Earth. As shown in Figure 3, it is started via 3D Ripper DX v1.8, so yellow 
characters "Ready to capture" appears on the top left corner of Google Earth software interface, which indicates normal operation of 3D Ripper DX v1.8. Then, we complete initialization setting of software environment.

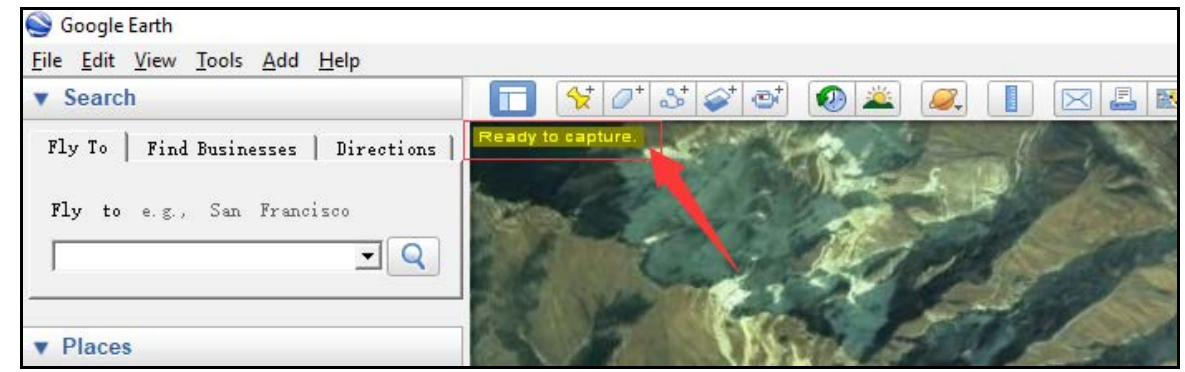

Fig. 3 Google Earth started via Ripper DX v1.8

\subsubsection{Extract data}

Taking Lu Tusi Governance located at Liancheng, Yongcheng, Lanzhou, Gansu for an example, as shown in Figure 4, we adjust it to a bird's-eye view and make the altitude of view be $6.55 \mathrm{~km}$, then we can see the terrain fluctuation image on Google Earth after remotely-sensing image.

After that, press "F12" on the keyboard, we can intercept 3D model in the current view, as shown in Figure 4. When red characters "Capturing frame, this can take up to 5 minutes... appear, it indicates success of model interception. Then, we save them to the storage catalog displaced by the flashed second column of yellow characters, namely self-defined "Frames (*.3DR) output directory" folder at the time of initialization setting of 3D Ripper DX v1.8.

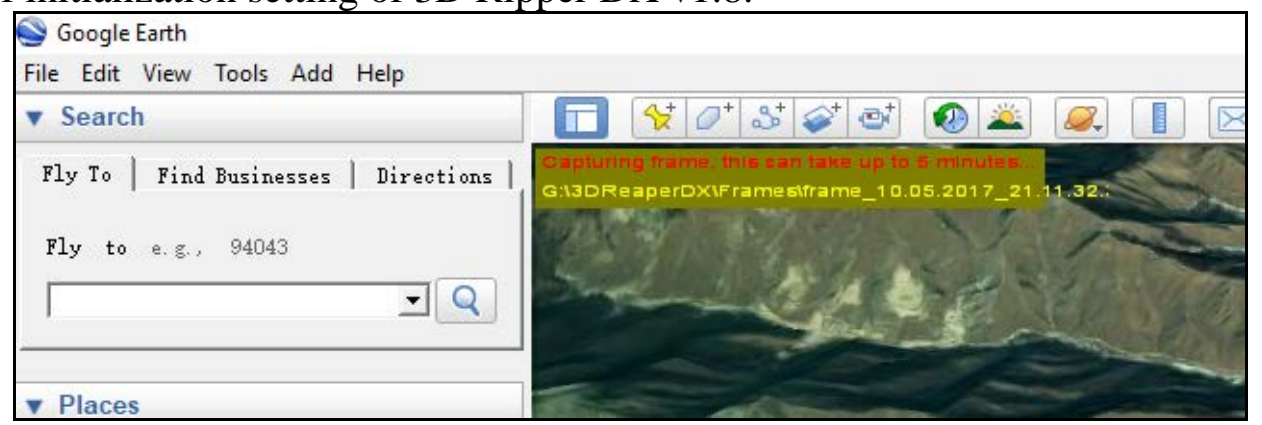

Fig. 4 Intercept 3D model after adjusted to be bird's-eye view

\section{Making and application of 3D terrain}

\subsection{Data imported to 3ds Max 2011}

As shown in Figure 5, open 3ds Max 2011, import *.3DR file in "Frames" fold to 3ds Max 2011 and set corresponding parameters of "3DR import options" according to Figure 6. Hereinto, "FOV (visual angle)" of "Reverce projection parameters" is based on height "60.00000" and "monitor aspect ratio" is 16:10 (1.6). For "Loading Method", select " 1 object=1 draw call", check "Remove double faces" and select "Remove faces drawn last "for "order". In "Options", check "Ignore ZENABLE=false meshes (usually HUD,2D elements)", "Ignore ZWRITEENABLE=false meshes (usually skybox)", "Ignore if RGBWRite is disabled (usually z-prepass) and "Enable texture display in viewports". 


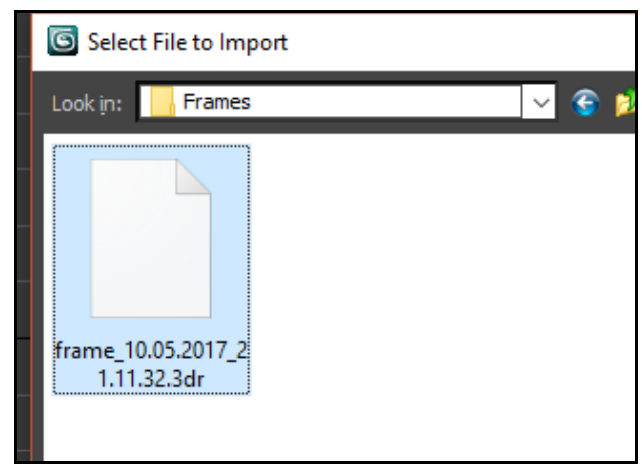

Fig. 5 Import 2dr file to 3ds Max 2011

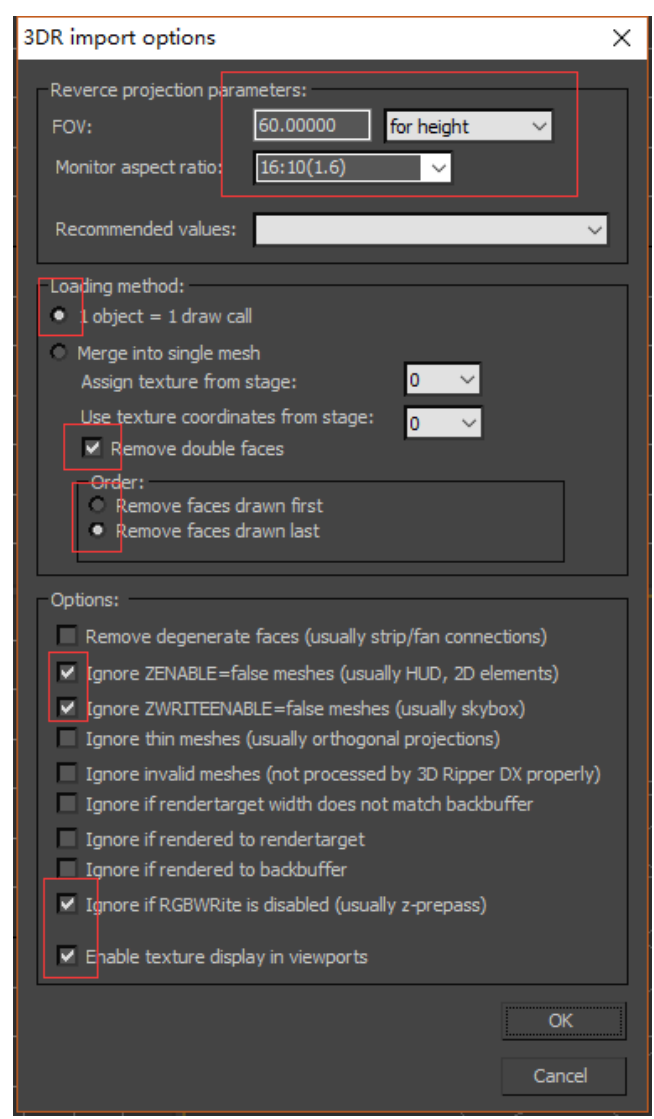

Fig. 6 Set up imported parameters of 3DR import options

All manuscripts must be in English, also the table and figure texts, otherwise we cannot publish your paper. Please keep a second copy of your manuscript in your office. When receiving the paper, we assume that the corresponding authors grant us the copyright to use the paper for the book or journal in question. Should authors use tables or figures from other Publications, they must ask the corresponding publishers to grant them the right to publish this material in their paper.

\subsection{Adjust and optimize imported data}

\subsubsection{Zoom-out model}

After import, the size of model data is mall, so we need adjust it to the visual size of current viewport. Use keyboard "Ctrl+A" combination key to check all the import models. As shown in Figure 7, click on "Select and Uniform Scale" key, then select "Transform Type-in..." function in "Edit" of menu bar and set up the multiplying power of "Offset: World" in the dialog box "Scale Transform Type...” popped up as shown in Figure 8 to be “10000000\%”. 


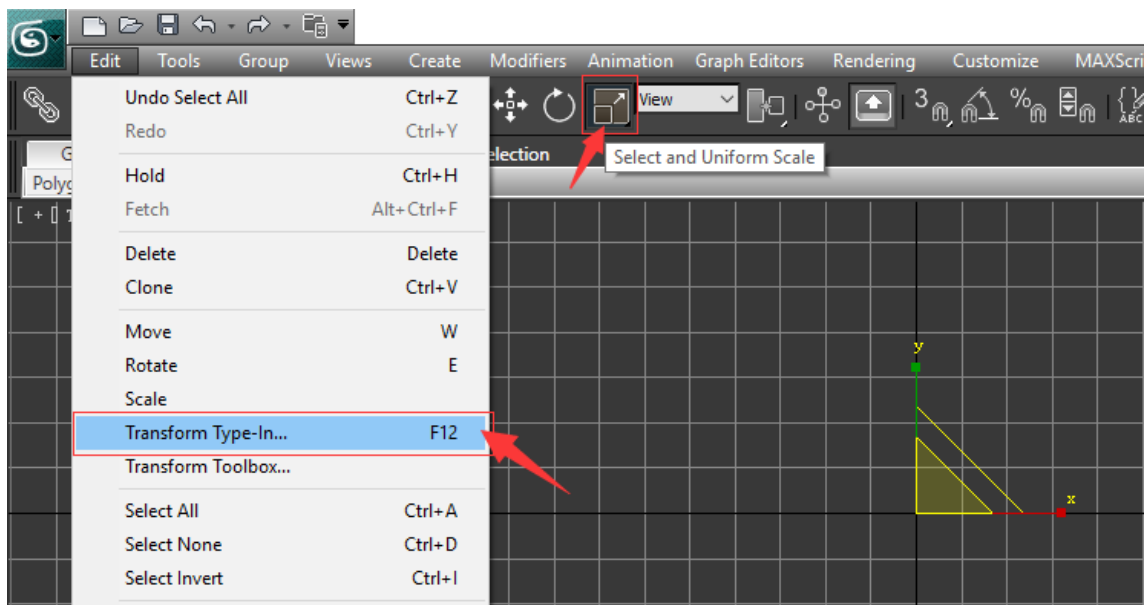

Fig. 7 Select model and zoom out evenly

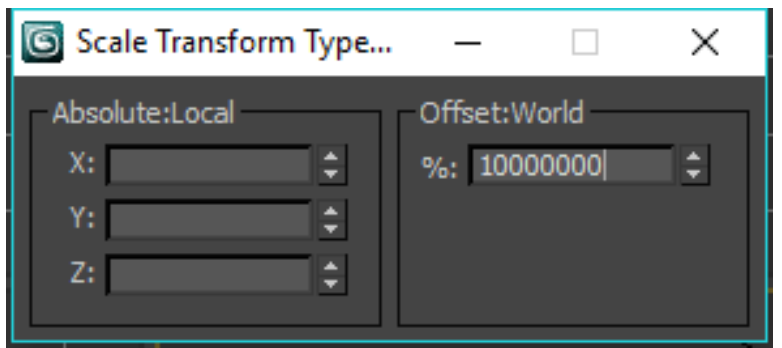

Fig. 8 Zoom-out conversion input

\subsubsection{Rotate model}

As shown in Figure 9, the size of model after zoom-out has been easy for operation, but it is not placed horizontally. At the moment, we need to take X-axis as the axle center to rotate model and adjust it until it is horizontal. Check all the imported model by using combination key "Ctrl+A", click on "Select and Rotate" button as shown in Figure 10, then select "Transform Type-in..." function in "Edit" of menu bar. As shown in Figure 11,the set up the rotating angle of X-axis in "Offset: World" of popped up "Rotate transform Typ..." dialog box according to model offset. According to the deviation angle, we set the rotating angle of X-axis in “Offset: World” as “-68”. The final effect after adjustment is shown in Figure 12.

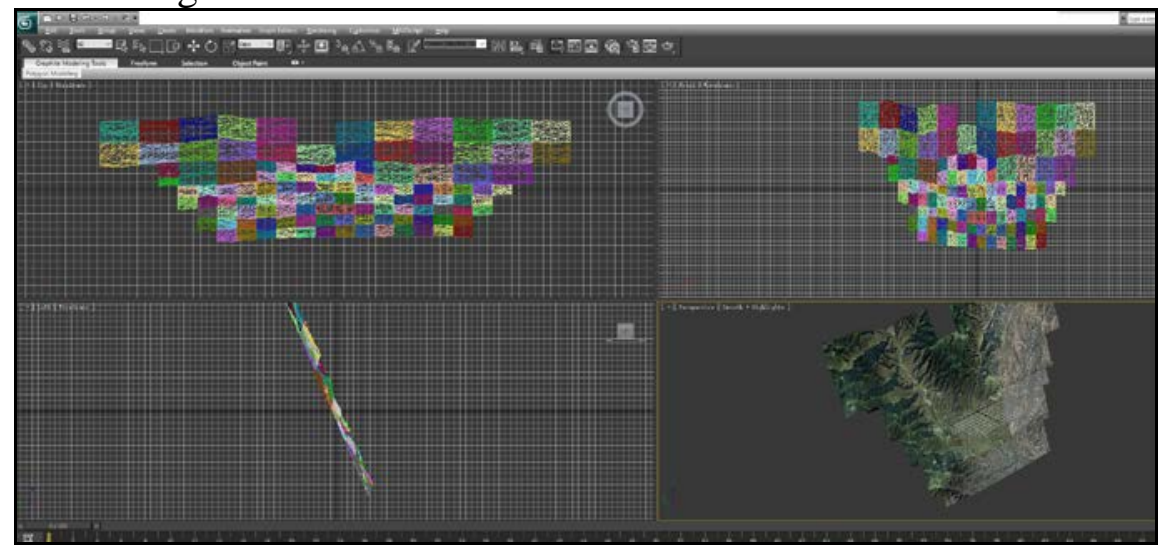

Fig. 9 Terrain model after zoom-out 


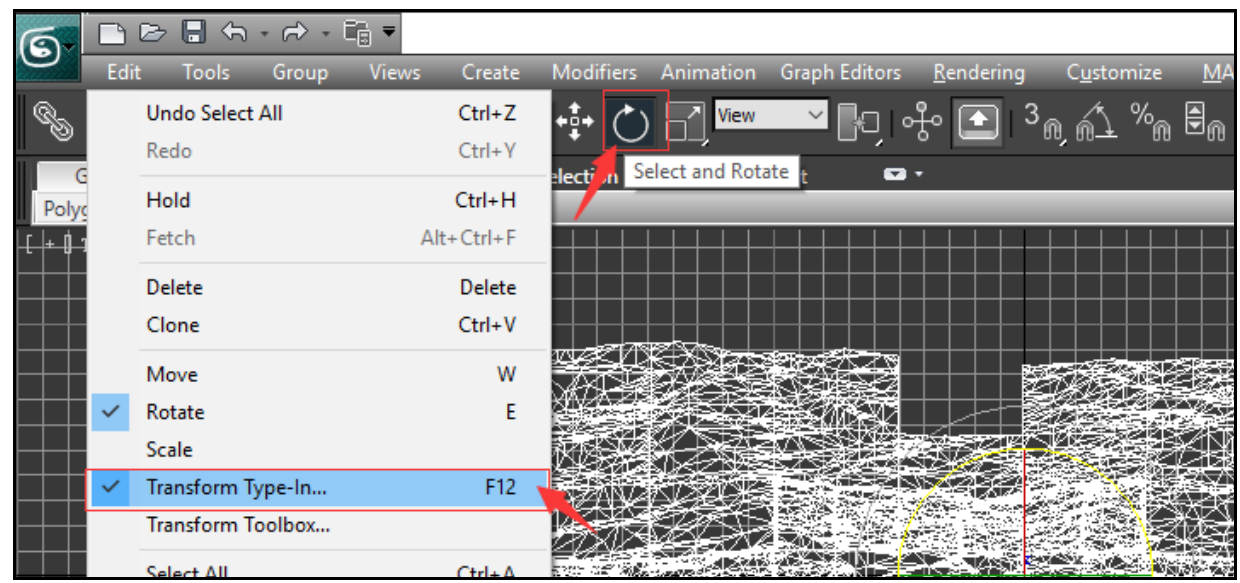

Fig. 10 Select and rotate model

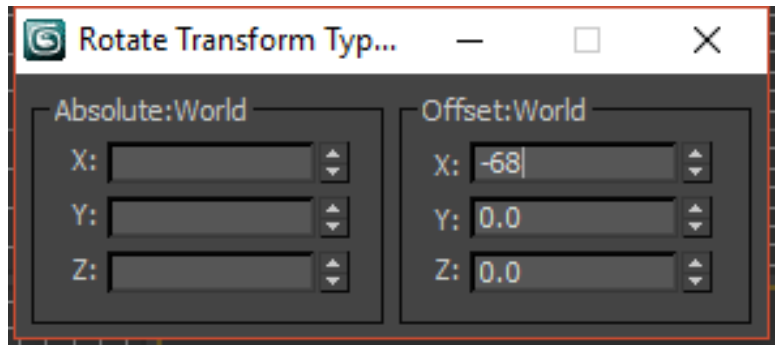

Fig. 11 Rotate model taking $\mathrm{X}$-axis as the axle center

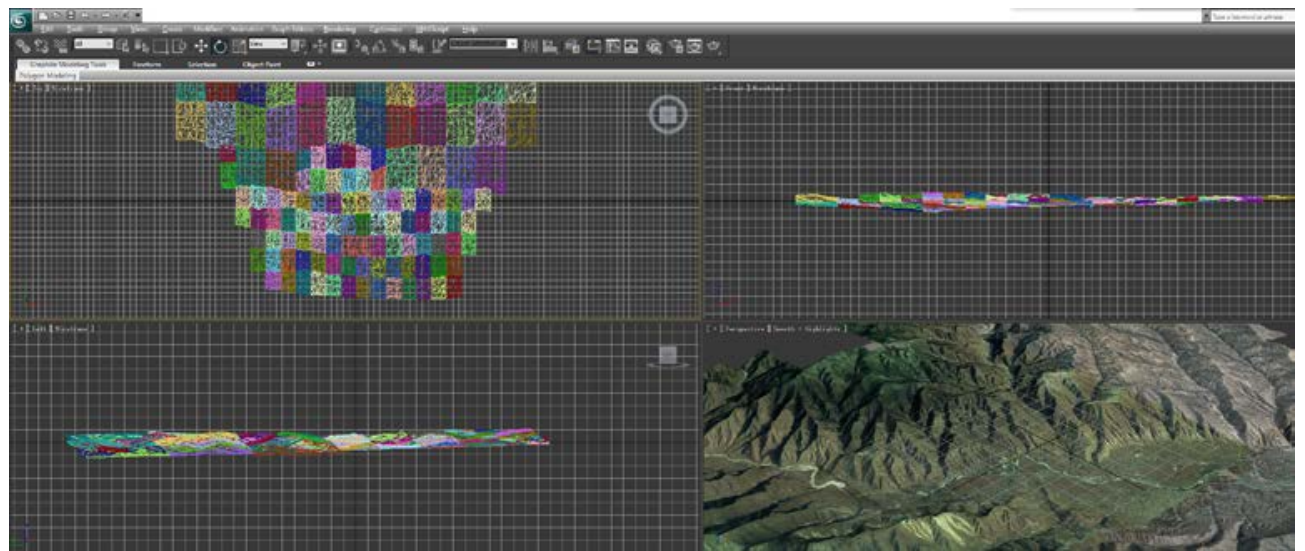

Fig. 12 Final effect after model adjustment

\subsubsection{Export model}

At this time, the model has met the use requirement, so we need to export it to OBJ format file. As shown in Figure 13, check "Texture coordinates" and "Normal" in "Geometry" option in "OBJ Export options" dialog box; check "Export materials" and "Create mat-library” in "Material” option; check "relative numbers" in "output" option and set the "Precision" as "6"; check "vertex", "normals" and "texture-coord" in "Optimize"; select "Preset" as "Deep Exploration" and then click on "Export”. 


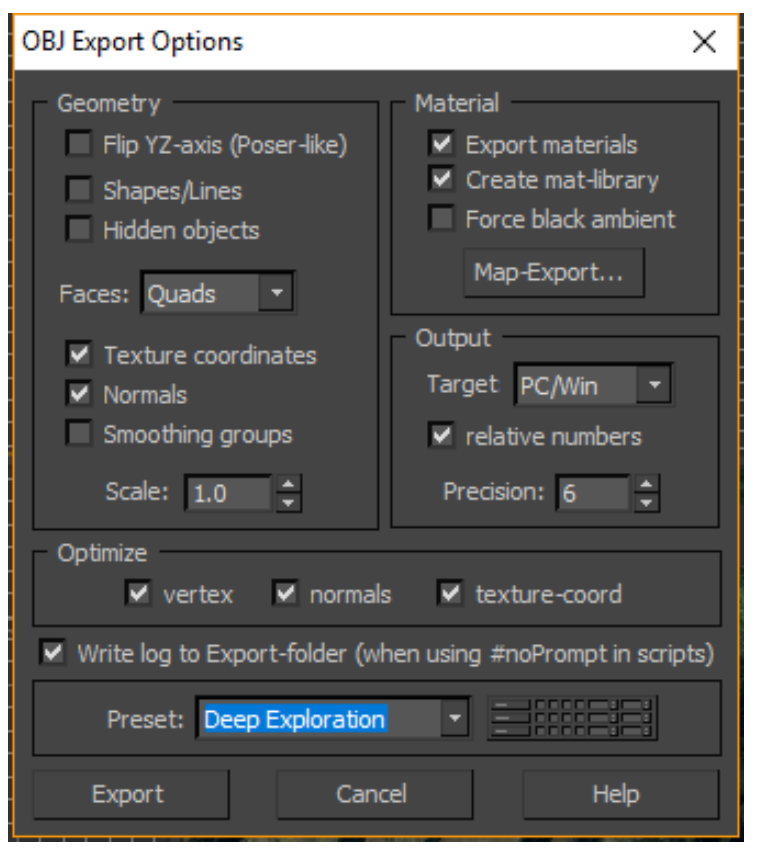

Fig. 13 OBJ export option

\subsubsection{Optimize model}

Start Deep Exploration 6.3, select to open OBJ file exported by using 3ds Max 2011 previously in the "File" menu. As shown in Figure 14, after clicking "Open", "Import Properties of Wavefront Object” dialog box pops up.Then, set up all the options as "YES” in "Common" and clicking "OK”.

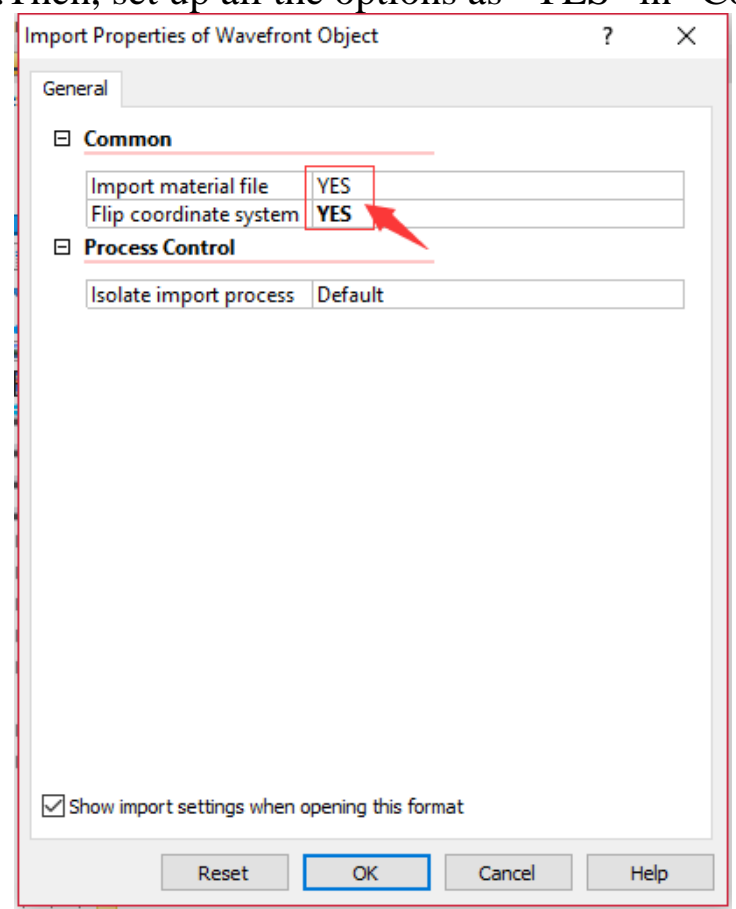

Fig. 14 Setting of import attribute

After opening OBJ file, as shown in Figure 15, select "Save" in the "file" and save the model as "C4D" format file. Then, start Cinema 4D R17 and open the saved C4D file. As shown in Figure 16, it is seen the model is still sorted according to the image data clipped by topographic map of DOM extracted from Google Earth at the beginning. Then, we can optimize and adjust 3D model of the terrain easily. 


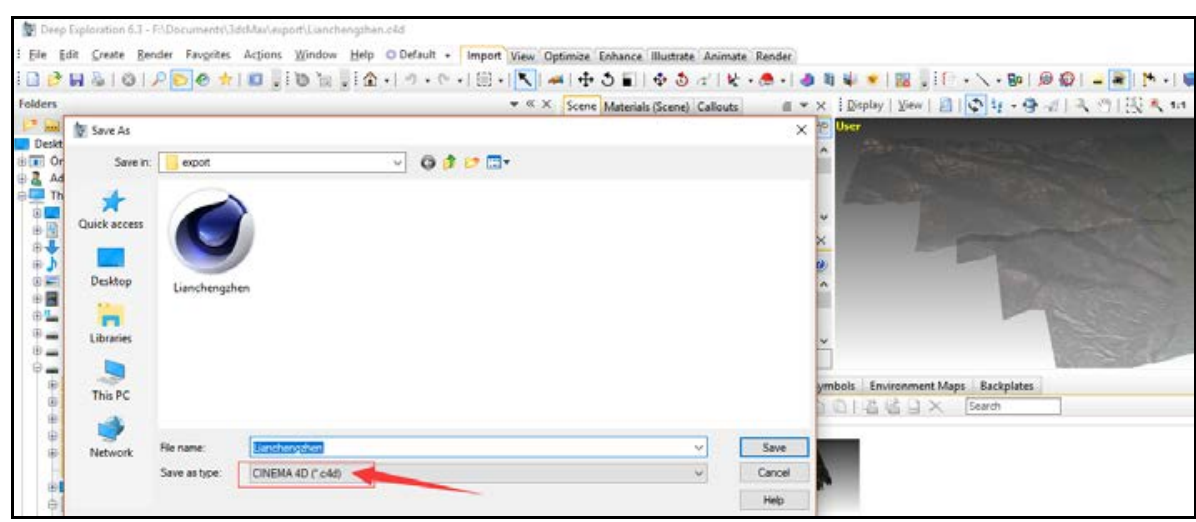

Fig. 15 Save as C4D format

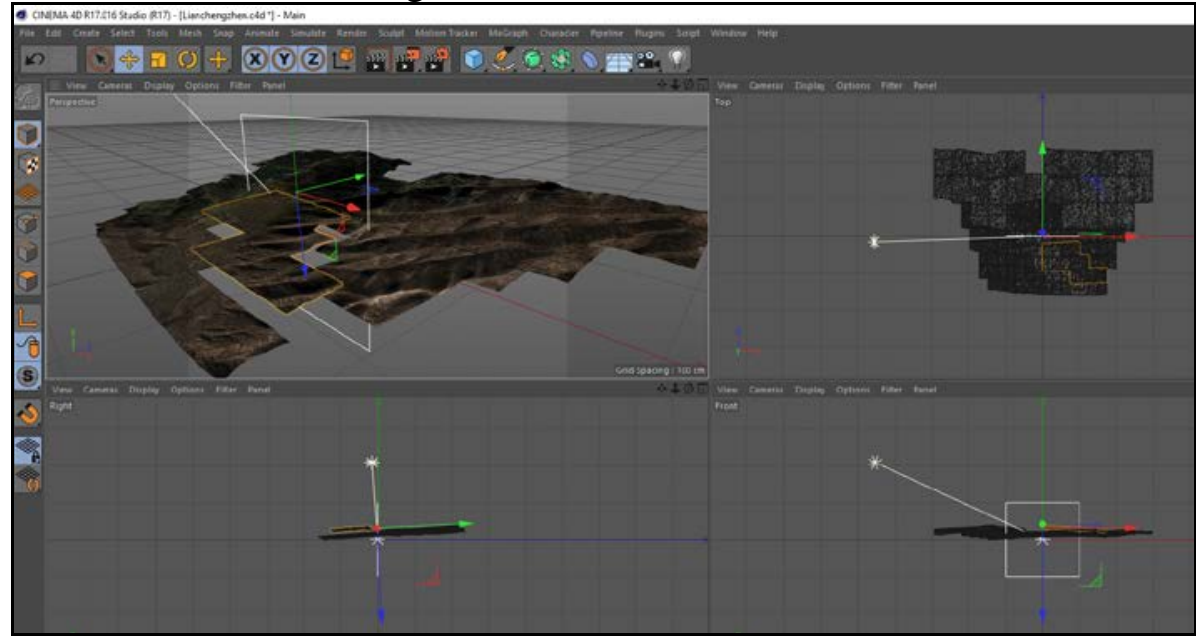

Fig. 16 Optimize 3D model of terrain in Cinema 4D R17

\section{Conclusions}

In Cinema 4D R17, not only can we optimize 3D model extracted from Google Earth Based on DOM, but also take Cinema 4D R17 as a bridge, utilize it to export standard FBX or OBJ file so as to expand DOM model utilization and utilization scope, make it be able to be loaded on most 3D software in the market and even imported to such game engines as Unity 3D and UE4. The method is used for making environmental modeling of 3D scene erection and virtual scene roam system of Lu Tusi Governance and Building Complex. Whatever making 3D roam or virtual interactive game, the application of the method can assist in 3D scene creation and display of real terrain of key cultural relics protection unit conveniently, rapidly and efficiently.

\section{References}

[1] Zhang Shifu. Discussion on High-Resolution Image Acquisition Method Based on Google Earth [J]. Electronic Technique, 2013,(12):16-18.

[2] Jiang Guocai. Interception and Assembly of Google Earth Image[J]. Guizhou Geology, 2010,(04):317-319+271.

[3] Tong Biao, Lu Chun, Wang Chunbo, Zhang Huihui. Research on DOM Updating Method based on Google Earth [J]. Journal of Liaoning Provincial College of Communications,2014,(05):32-35.

[4] Zhang Jianqing, et al. Pjotogrammetry[M]. Wuhan University Press,2003. 